\title{
Grupo educativo de cirurgia cardíaca em um hospital universitário: impacto psicológico
}

\author{
Cardiac surgery educational group in a university \\ hospital: psychological impact
}

\author{
Jeanny Joana Rodrigues Alves de SANTANA \\ Luan Flávia Barufi FERNANDES \\ Carla Rodrigues ZANIN ${ }^{3}$ \\ Christiane Maia WAETEMAN ${ }^{3}$ \\ Marcelo SOARES ${ }^{4}$
}

\begin{abstract}
Resumo
Este estudo avaliou o impacto psicológico de um grupo educativo de cirurgia cardíaca em pacientes de um hospital universitário. Participaram 73 sujeitos, dos quais 62\% eram homens. A média de idade foi 52 anos, desvio-padrão foi 16. Os pacientes foram submetidos a uma sessão multidisciplinar com exposição dialogada sobre cardiopatias, procedimento cirúrgico e sintomas psicológicos. Os resultados mostraram que, para poucos pacientes (36\%), o grupo ajudou a esclarecer dúvidas sobre o tratamento, demonstrando a necessidade de ajustes nas estratégias didáticas para melhor compreensão dos conteúdos. A análise das emoções percebidas após participação no programa revelou que 70\% responderam estar esperançosos. A contraposição entre "esperança" e "tranquilidade" foi uma resposta emocional esperada frente à vivência da cirurgia, avaliada como uma condição de ameaça ambiental. Conclui-se que o grupo educativo foi benéfico para o ajustamento psicossocial do paciente à cirurgia. Entretanto, maior eficácia será alcançada mediante aperfeiçoamento do método de atuação e inclusão de um programa interdisciplinar estruturado para controle do stress.
\end{abstract}

Unitermos: Cirurgia torácica. Impacto psicossocial. Instrução em grupo.

\footnotetext{
Abstract

This study assessed the psychological impact of an educational group for cardiac surgery patients in a university hospital. Seventy-three subjects took part in the study-62\% were men with an average age of 52 (standard deviation $=16$ ), subjected to a multidisciplinary session including discussion about heart disease, surgical procedures and psychological symptoms. The results showed that for a few patients (36\%), the group helped to clarify questions about the treatment, demonstrating the need for adjustments in teaching strategies for a better

\section{$\operatorname{crut}$}

1 Universidade de São Paulo, Faculdade de Filosofia, Ciências e Letras de Ribeirão Preto, Departamento de Psicologia e Educação, Pós-Graduação em Psicobiologia. Av. Bandeirantes, 3900, Bloco 6, Sala 34, Monte Alegre, 14040-901, Ribeirão Preto, SP, Brasil. Correspondência para/Correspondence to: J.J.R.A. SANTANA. E-mail: <jeanny.santana@terra.com.br>.

2 Faculdade de Medicina de São José do Rio Preto, Hospital de Base, Serviço de Psicologia. São José do Rio Preto, SP, Brasil.

3 Faculdade de Medicina de São José do Rio Preto, Programa de Aprimoramento em Psicologia da Saúde. São José do Rio Preto, SP, Brasil.

4 Faculdade de Medicina de São José do Rio Preto, Hospital de Base. São José do Rio Preto, SP, Brasil.

Agradecimentos: ao Prof. Dr. José Antônio Cordeiro, pelo auxílio na análise dos dados e ao Prof. Dr. Fernando Batigália, pela assessoria científica.
} 
understanding of the content. The analysis of emotions perceived after participation in the program showed that $70 \%$ were said to be hopeful. The contrast between "hope" and "tranquility" was an expected emotional response given the experience of surgery, evaluated as an environmental threat condition. It was concluded that the educational group was beneficial to the patients' psychosocial adjustment to surgery. However, greater effectiveness will be achieved by improving the performance method and the inclusion of an interdisciplinary program designed to control stress.

Uniterms: Thoracic surgery. Psychosocial impact. Group instruction.

A cirurgia cardíaca é uma modalidade de tratamento para diferentes cardiopatias que gera sofrimento para o indivíduo em muitos aspectos. No âmbito biológico, o paciente está suscetível a sensações de dor, infecções, intervenções invasivas e risco de morte. No campo social, o paciente se afasta temporariamente do convívio com os amigos e parentes pelo tempo de internação hospitalar, tem limitada sua autonomia e diminui ou extingue as atividades laborais. Quanto à condição psicológica, geralmente o paciente apresenta sintomas de ansiedade, depressão e tem expectativas negativas sobre o futuro (Medeiros, 2002; Neme \& Rodrigues, 2003; Romano, 2001; Souza, 2004). O enfrentamento da situação consiste na busca por respostas adaptativas e no manejo das experiências, estados emocionais e comportamentos causados pelo stress. Essas estratégias referem-se a padrões cognitivos e comportamentais que são empregados para manter o equilíbrio diante das especificidades da situação de adoecimento (Lazarus, 1993).

A psicologia da saúde - especialmente a psicologia aplicada à cardiologia - fundamentou, ao longo de anos, uma enorme gama de conhecimentos para respaldar a prática profissional em situações estressoras como a sofrida por pacientes de cirurgia cardíaca. 0 resultado deste trabalho é o desenvolvimento de teorias, métodos e técnicas de intervenção como, por exemplo, o treino psicológico para controle do stress (Lipp, 2001), análise funcional de contingências no hospital (Gorayeb \& Guerrelhas, 2003), intervenção multimodal e multidisciplinar para reabilitação pós-enfarte do miocárdio (Fernandes \& Mclntyre, 2006), estudos sobre adesão ao tratamento (Alfonso \& Abalo, 2004) e suporte social aos pacientes (Resende, Cunha, Silva \& Sousa, 2007), pesquisas sobre preditores comportamentais de saúde (López, García \& Dresch, 2006; Ribeiro, 2004), desenvolvimento de instrumentos para avaliação de estratégias de enfrentamento (Seidl,Tróccoli \&Zannon, 2001), investigação sobre problemas psicológicos associados ao transplante de órgãos (San Gregorio, Rodríguez \&

32 Rodríguez, 2005), entre outros temas.
As atribuições do psicólogo em uma equipe de cirurgia são: compreender o comportamento no contexto da saúde e doença, determinar e conhecer as condições comportamentais e emocionais do paciente, oferecer assistência psicológica a ele, executar pesquisas e participar de atividades de ensino. Com a evolução das concepções de saúde-doença o papel do psicólogo passou a incluir o atendimento aos familiares e realização de intervenções educativas (Angerami-Camon, 1996; Kerbauy, 2002).

A educação em saúde, entendida aqui como a prática voltada para o desenvolvimento de habilidades individuais e coletivas em busca da melhoria da qualidade de vida, é estruturada a partir de uma tendência pedagógica. Se a diretriz for tradicional, haverá predominância da exposição oral dos conteúdos, com uma sequência fixa, predeterminada e sem relação com o cotidiano do sujeito. A abordagem crítica valoriza a problematização, o conhecimento da realidade, a integração entre os participantes para criar soluções e, assim, desenvolver a autonomia (Pereira, 2003). A consequência de uma intervenção educativa em cirurgia cardíaca nos moldes da abordagem crítica é o aprendizado, pelo paciente, de habilidades de análise e compreensão da situação que ele vivencia. Este é um elemento favorecedor da diminuição do impacto negativo do tratamento na vida do indivíduo. Há evidências empíricas de que a prática educativa proporciona minimização dos sintomas de ansiedade e depressão, influencia positivamente a recuperação clínica após a cirurgia e interfere no prognóstico e evolução do paciente (Neme \& Rodrigues, 2003).

É recomendável que o trabalho educativo seja planejado pela equipe interdisciplinar em união com as pessoas atendidas. Inicialmente, são identificadas as características da população e definidos os objetivos da atividade. Posteriormente, o método é estabelecido pela integração de conhecimentos entre os profissionais e os resultados avaliados periodicamente, para que a prática seja sempre otimizada. Neste processo, o psicó- 
logo utiliza instrumentos para investigar a demanda e analisar os resultados, emprega ações que beneficiem a coesão do grupo de profissionais, bem como a comunicação genuína de ideias, e aplica técnicas psicológicas para que o trabalho com o paciente seja realmente eficaz. Dada a complexidade do papel do psicólogo na equipe interdisciplinar, ele precisa ser adequadamente capacitado para justificar com coerência suas propostas. Por isso, deve lançar mão de um vasto referencial teórico, como o da Psicologia da Saúde, Psicologia Institucional, Psicologia dos Grupos, Teorias e Técnicas Psicoterápicas, Teorias da Aprendizagem, Psicobiologia e Ética (Granada, 2004; Scorsafava, 2004; Tonetto \& Gomes, 2007).

Os resultados de um programa educativo com pacientes de cirurgia cardíaca envolvem a qualidade da integração entre os profissionais e a noção que a equipe interdisciplinar tem dos limites da intervenção. Uma ação integrada entre disciplinas é caracterizada pela comunicação de ideias e associação de procedimentos. Se estes fatores não estão presentes, não podem ser considerados ações interdisciplinares, mas práticas profissionais isoladas (Granada, 2004). A prática de educação em saúde é limitada, pois somente a transmissão de informações não é o suficiente para modificar comportamentos de forma duradoura. Na análise comportamental considera-se que o indivíduo deve estar exposto contínua e exaustivamente às contingências ambientais, interpessoais e fisiológicas favorecedoras do desenvolvimento e fortalecimento de categorias específicas de respostas como, por exemplo, cuidados com o próprio corpo, uso de estratégias mais adequadas de enfrentamento à doença e adesão ao tratamento medicamentoso (Catania, 1999).

Reconhecendo que um programa educativo em cirurgia cardíaca possui tanto benefícios quanto limitações, é fundamental identificar estes elementos para que a prática de atuação seja aperfeiçoada. Neste sentido, o objetivo deste estudo foi avaliar o impacto psicológico de um grupo educativo de cirurgia cardíaca em pacientes internados na enfermaria de um hospital universitário do interior de São Paulo.

\section{Método}

\section{Participantes}

Foram colaboradores deste estudo 73 pacientes internados no setor de cirurgia cardíaca de um hospital do interior de São Paulo que participaram de um grupo educativo antes e/ou após a cirurgia. Do total de sujeitos, $62 \%$ eram do sexo masculino e 38\% feminino. A média de idade foi 52 anos (desvio-padrão=16). Foram incluídos pacientes de ambos os sexos, sem restrição de idade, escolaridade, nível socioeconômico, cor, religião e estado civil. Foram excluídos do estudo aqueles que se recusaram a participar da pesquisa ou apresentaram comprometimento neurológico, psiquiátrico, alterações de consciência (coma) ou outro comprometimento físico (pacientes com ventilação mecânica).

\section{Instrumentos}

Os instrumentos utilizados foram uma ficha de identificação elaborada pelos pesquisadores, contendo dados pessoais e condições socioeconômicas, e uma entrevista estruturada para avaliação do impacto psicológico do grupo educativo de cirurgia cardíaca, ambos elaborados pelos pesquisadores. A entrevista consistiu de quatro questões fechadas, com suas respectivas opções de resposta: a) motivação dos pacientes para participar do grupo (esclarecer dúvidas, conhecer pessoas com a mesma doença, dar e receber apoio social); b) o quanto o grupo ajudou a enfrentar a cirurgia (nada, um pouco, mais ou menos, bastante e muito); c) fatores de auxílio do grupo (aceitar melhor a doença, diminuir o medo, ter mais coragem e confiança, esclarecer dúvidas sobre a doença, sobre o tratamento e sobre o sucesso da cirurgia, encontrar apoio de outras pessoas, mudar a concepção prévia sobre a doença e cirurgia e melhorar o humor); d) emoções percebidas após participação no grupo (medo, preocupação, tristeza, tédio, raiva, confusão, nervosismo, angústia, sentir-se mal após participar, animação, felicidade, esperança, tranquilidade, alívio, empatia). Os participantes poderiam assinalar mais de uma alternativa em cada fator, exceto para a questão "b", cujas alternativas eram mutuamente excludentes.

\section{Procedimentos}

Os pacientes, tanto na condição pré quanto na pós-operatória, foram convidados a participar do grupo coordenado pela psicóloga, pela enfermeira e pela nutricionista. $\bigcirc$ programa foi estruturado com sessão única de 60 minutos, bissemanal, com exposição 
dialogada, utilização de recurso visual (data show) e aberto a, no máximo, quinze pessoas (além dos pacientes, os familiares também puderam participar da atividade). Os profissionais incentivaram a elaboração de perguntas sobre os assuntos abordados.

Cada módulo de vinte minutos seguiu roteiro conforme a disciplina que o fundamentou. Inicialmente, a psicóloga debateu com os pacientes sobre a cirurgia (natureza do procedimento e recuperação), fatores de risco (tabagismo, hábitos alimentares e exercícios físicos), comportamentos relacionados ao procedimento (ansiedade, depressão) e conversou sobre modificação de hábitos em prol de uma vida saudável. Em seguida, a enfermeira dialogou sobre cuidados com higiene e prevenção de infecção. Finalmente, a nutricionista discutiu com os participantes sobre hábitos alimentares saudáveis para doenças crônico-degenerativas relacionadas às cardiopatias como diabetes, dislipidemia e hipertensão. As profissionais buscaram seguir este roteiro para abranger o maior número de temas sobre o tratamento. Entretanto, ele não era cumprido rigorosamente, devido à restrição de tempo e também porque a prioridade estava centrada no diálogo e no esclarecimento de dúvidas. A experiência dos pacientes, verificada por meio dos depoimentos e troca de ideias, era referência para a construção de conceitos, e os exemplos abordados por eles eram propícios para relacionar a teoria à prática vivenciada no cotidiano.

Após o programa educativo, os objetivos da pesquisa foram apresentados. Os pacientes que aceitaram o convite para participar da pesquisa assinaram o Termo de Consentimento Livre e Esclarecido em duas vias e receberam uma cópia. Este documento foi elaborado conforme os princípios da Resolução 196/96 do Conselho Nacional de Saúde (CNS) do Ministério da Saúde, contendo dados de identificação dos pesquisadores e dos sujeitos, objetivos e procedimentos da pesquisa e garantias quanto ao sigilo e anonimato do participante. Em seguida, os pacientes responderam aos instrumentos de coleta de dados. Como cada grupo continha no máximo quinze pessoas e, destas, somente algumas eram pacientes, a coleta de dados se estendeu por um ano para alcançar o tamanho adequado da amostra. Apesar de participarem juntos da intervenção educativa, somente os pacientes (e não os familiares) foram sujeitos da pesquisa e responderam aos instru34 mentos.
Os dados foram analisados quantitativamente, apresentados em estimativa de percentual (intervalo de confiança 95\%) e confrontados com 50\% por meio do teste exato para uma proporção. Foi adotado o nível de significância $\alpha \leq 0,05$. Realizou-se a estatística descritiva (frequências, porcentagens, médias, medianas, desvio-padrão) dos dados sociodemográficos da amostra e das seguintes questões avaliadas: "O quanto o grupo ajudou a enfrentar a cirurgia", "Fatores de auxílio do grupo" e "Motivação dos pacientes para participar do grupo". A análise dos componentes principais foi aplicada para a questão "emoções percebidas após participação no grupo", com o objetivo de analisar as informações a partir da semelhança entre indivíduos e definir uma topologia das variáveis, isto é, reduzir as variáveis originais para um número pequeno de índices (componentes principais) e, assim, conhecer como os subgrupos estavam correlacionados entre si (Crivisqui, 1999).

\section{Resultados e Discussão}

Conforme mencionado, o impacto psicológico da intervenção educativa nos pacientes hospitalizados foi avaliado mediante as seguintes questões: a) motivação dos pacientes para participar do grupo; b) o quanto o grupo ajudou a enfrentar a cirurgia; c) fatores de auxílio do grupo; d) emoções percebidas após participação no grupo.

No item "a" (motivação dos pacientes para participar do grupo), verificou-se que uma minoria teve como motivação participar "para ajudar os outros"(15\%), "para conhecer pessoas com a mesma doença" (22\%) e "para obter apoio de outras pessoas" (23\%) ( $p=0,000)$, ou seja, uma minoria esperava receber ou oferecer suporte social. O psicólogo deve discutir com a equipe sobre meios para incentivar a interação dos participantes e realizar um treino de habilidades com eles para que identifiquem, obtenham e mantenham o suporte social, elemento promotor de saúde (Resende et al., 2007).

Por outro lado, foi estimado que a maioria dos participantes $(74 \%, p=0,000)$ estava motivada a participar do grupo para tirar dúvidas e obter informações sobre a cirurgia. Ao auxiliar o paciente a compreender a situação vivenciada, é oferecido um clima de confiança entre ele 
e os profissionais, o que favorece a adesão ao tratamento no período pós-operatório e, mais importante que isso, auxilia na identificação de fatores relacionados ao indivíduo, à equipe e à instituição que dificultam a adesão (Benute et al., 2001).

No item "b" (o quanto o grupo ajudou a enfrentar a vivência da cirurgia), verificou-se que a maioria (58\%, $p \leq 0,05)$ avaliou o grupo positivamente, considerando-o muito importante para o auxílio no enfrentamento da situação estressora. A avaliação positiva da intervenção pode resultar em benefícios como menor duração da internação e melhora da capacidade funcional (Neme \& Rodrigues, 2003). Estudos longitudinais devem ser feitos para avaliar os efeitos da atividade nestas variáveis.

No item "c" (fatores de auxílio), uma minoria respondeu que o grupo ajudou "a esclarecer dúvidas sobre sucesso da cirurgia" (36\%; $p=0,019)$, "a diminuir o medo" (36\%; $p=0,019)$, "a aceitar melhor a doença" (33\%; $p=0,005)$, "a mudar a ideia que tinha sobre a doença" (27\%; $p=0,000)$, "a encontrar apoio de outras pessoas" (pacientes e acompanhantes) (26\%; $p=0,000)$ e"melhorar o humor" (diminuir sentimentos de tristeza e depressão) (22\%; $p=0,000)$. A alternativa "o grupo não ajudou" não foi assinalada por qualquer participante $(0 \%$; $p=0,000)$. Provavelmente, a baixa frequência de respostas às alternativas do item "fatores de auxílio do grupo" deve-se a três motivos principais, citados a seguir.

O primeiro deles diz respeito às falhas na construção do instrumento. A pergunta feita aos participantes - "Em que fatores o grupo educativo te ajudou, neste momento de sua vida?" - pressupôs que o programa educativo auxiliou o paciente a enfrentar a vivência da cirurgia em pelo menos um dos aspectos indicados nas alternativas (aceitar a doença, diminuir o medo, ter mais confiança no tratamento etc.). Somente a alternativa "o grupo não ajudou" contemplou um possível resultado negativo da intervenção. As regras de construção de instrumentos de pesquisas nas ciências humanas estabelecem que as perguntas devem ser elaboradas sem prévio julgamento das respostas dos participantes (Cruz\& Ribeiro, 2003).

O segundo equívoco identificado neste item é que os pesquisadores foram os coordenadores da intervenção e isto, por si só, caracteriza-se como um viés de pesquisa. Provavelmente, o participante se viu constrangido em atribuir valor negativo ao programa, mesmo que esta fosse a resposta de sua escolha. Infelizmente, a falta de rigor na elaboração e aplicação do instrumento de coleta de dados é um forte elemento de limitação deste estudo.

Em terceiro lugar, a baixa frequência de respostas às alternativas do item "fatores de auxílio do grupo" sinaliza que, no formato que foi constituído neste estudo, o programa educativo não possui impacto significativo em variáveis complexas como aceitação da doença e diminuição do medo. É recomendável o planejamento de uma intervenção que integre as ações dos profissionais para atender às necessidades do paciente de modo global. Não basta que cada profissional contribua com seu corpo de conhecimentos para chegar a um objetivo comum. O ideal é buscar uma unidade conceitual materializada na criação de estratégias efetivas para a promoção de saúde e controle do estressor (Granada, 2004).

O fato de o grupo educativo não ter ajudado a esclarecer dúvidas dos pacientes sobre o tratamento indica que mudanças urgentes e necessárias precisam ser empreendidas para o alcance deste objetivo. Uma primeira medida a ser tomada é o ajuste nas estratégias didáticas (emprego de métodos de interação grupal e uso de material didático alternativo). A realização de um trabalho informativo, intensivo, executado pelos membros da equipe ao longo da internação, e não somente durante a sessão educativa, é outro procedimento a ser adotado. As sugestões são coerentes com a teoria da aprendizagem social de Bandura, que assegura que a educação em saúde é influenciada e determinada pelo ambiente e cultura e, por isto, só é efetiva se realizada de forma contínua e gradativa (Bandura, Azzi \& Polydoro, 2008).

A ineficácia do programa em sanar dúvidas dos pacientes chama a atenção para a definição dos objetivos da atividade, que devem ir além da transmissão de informações. Conforme o paradigma da Promoção de Saúde, o coordenador de uma intervenção educativa deve compreender que o indivíduo é capaz de avaliar a realidade que vivencia e de desenvolver habilidades de resolução de problemas de adoecimento, ou seja, ele pode autogerir sua saúde. Neste sentido, a tarefa dos profissionais não é meramente repassar informações, mas também planejar ações estimuladoras do 
autoconhecimento e da autogestão, para que o paciente assuma o controle e a responsabilidade por sua própria saúde (Baremblitt, 2002; Coates, 1999). Para tanto, o planejamento da atuação deve fugir das "receitas de comportamento" e do discurso de culpabilidade do tipo "Se ingerir muito sal, a pressão vai subir e pode dar infarto", e mostrar a possibilidade de viver prazerosamente e ter hábitos saudáveis de vida. A organização de um programa com estes preceitos exige delegação de novas tarefas aos profissionais e implica recomposição das relações de trabalho. Infelizmente, a consequência esperada é a resistência de membros da equipe às mudanças, pois elas alteram uma organização vigente (Coates, 1999; Granada, 2004; Maia, 2006).

Em uma situação de resistência, o psicólogo tem a tarefa de analisar a estrutura institucional, questionar os objetivos das práticas, relacionar as ações dos profissionais com as concepções culturais de saúde e políticas públicas, identificar processos bloqueadores de mudanças (como a burocratização e a rigidez hierárquica do sistema de gestão), levantar dados sobre as contradições institucionais (por exemplo, discurso de integração de disciplinas, embora as práticas sejam isoladas) e envolver a equipe em um movimento institucionalista com o compromisso de implementar processos de mudança buscando restaurar o lugar do paciente como sujeito de sua própria vida, capaz de realizar a autoanálise e a autogestão da doença (Maia, 2006).

A análise do item "d" (emoções percebidas após participação no grupo) mostrou uma pequena estimativa para emoções de ansiedade (medo e preocupação) e depressão (tristeza, desânimo, desesperança), apontando para a consideração de que a intervenção não teve impacto negativo nas respostas emocionais dos pacientes (Tabela 1).

Por outro lado, uma minoria relatou presença de emoções de felicidade e alívio. Conjectura-se que obter um sentimento de felicidade não seja a expectativa do paciente que participa deste tipo de grupo, pois ele pretende, em primeira instância, conhecer o procedimento cirúrgico, para tomar ciência das contingências às quais está exposto. Além disso, o alcance da felicidade depende de outros fatores, como ganho de reforçadores positivos (por exemplo, resgatar a saúde física), o que não condiz com a vivência de submissão à cirurgia
Tabela 1.Emoções percebidas após participação no grupo. São José do Rio Preto (SP), 2007.

\begin{tabular}{lcc}
\hline Emoções percebidas após participação no grupo & $\mathrm{n}$ & $\%$ \\
\hline Esperança* $^{*}$ & 51 & 70 \\
Tranquilidade** & 38 & 52 \\
Ânimo** & 33 & 45 \\
Sem dúvidas** & 28 & 38 \\
Felicidade* & 25 & 34 \\
Alívio* $^{* *}$ & 23 & 32 \\
Empatia* $_{\text {Preocupação* }}^{*}$ & 22 & 30 \\
Medo* & 7 & 10 \\
Tristeza* & 5 & 7 \\
Nervosismo* & 2 & 3 \\
Desânimo* & 2 & 2 \\
Com dúvidas" & 1 & 1 \\
Tédio* & 1 & 1 \\
Desesperança* & 1 & 1 \\
Raiva* & 0 & 0 \\
Sentiu mal" & 0 & 0 \\
\hline
\end{tabular}

$" p<0,05 ; " " p>0,05$.

cardíaca, um evento altamente aversivo para os pacientes. É preciso levar em conta a influência do sistema nervoso autônomo no comportamento emocional de ansiedade (Catania, 1999). No caso da cirurgia cardíaca, que representa ameaça e risco à vida do paciente, frequentemente ocorre ação simpática e parassimpática, enquanto permanecer exposto a esta contingência aversiva. Cabe ao psicólogo auxiliar o paciente (com uso de técnicas específicas) a desenvolver habilidades para ajustamento satisfatório a esta contingência.

Cumprindo uma necessidade de análise pormenorizada da questão "emoções percebidas após participação no grupo", com intenção de verificar a associação entre os itens deste tema, utilizou-se um recurso da estatística, que é o Método de Análise dos Componentes Principais (Tabela 2).

Verifica-se que, com 36\% da variação total, o primeiro fator ponderou para aspectos positivos. Cada um destes aspectos é representado aqui com suas respectivas cargas fatoriais, que indicam a correlação entre aquele item da variável e o fator total, sendo estes valores variando entre-1 e 1: alívio $(0,44)$; esclarecimento de dúvidas $(0,44)$; tranquilidade $(0,42)$; ânimo $(0,39)$. Já no segundo fator, com $14 \%$ da variação total, foram contrapostos sentimentos de esperança $(-0,71)$ e tranquilidade $(0,45)$, ou seja, um grupo de pacientes estava tranquilo, 
Tabela 2. Análise dos componentes principais das emoções percebidas pelos pacientes após participação no grupo educativo de cirurgia cardíaca. São José do Rio Preto (SP), 2007.

\begin{tabular}{|c|c|c|c|c|c|}
\hline Eigenvalue & 0,66209 & 0,26045 & 0,20744 & 0,18145 & 0,13450 \\
\hline \% variância & 0,35500 & 0,14000 & 0,11100 & 0,09700 & 0,07200 \\
\hline$\%$ acumulada & 0,35500 & 0,49400 & 0,60600 & 0,70300 & 0,77500 \\
\hline \multirow{2}{*}{ Variáveis } & \multicolumn{5}{|c|}{ Componentes } \\
\hline & 1 & 2 & 3 & 4 & 5 \\
\hline Esperança & 0,221 & $-0,710$ & 0,141 & $-0,144$ & 0,123 \\
\hline Tranquilidade & 0,423 & 0,448 & $-0,189$ & 0,487 & $-0,046$ \\
\hline Ânimo & 0,390 & $-0,314$ & $-0,604$ & 0,119 & 0,272 \\
\hline Sem dúvidas & 0,440 & 0,042 & 0,296 & $-0,191$ & $-0,625$ \\
\hline Felicidade & 0,301 & 0,347 & $-0,177$ & $-0,801$ & 0,241 \\
\hline Alívio & 0,442 & $-0,193$ & 0,151 & 0,080 & $-0,208$ \\
\hline Empatia & 0,344 & 0,145 & 0,554 & 0,181 & 0,600 \\
\hline Preocupação & $-0,125$ & 0,041 & 0,303 & $-0,060$ & 0,225 \\
\hline Medo & $-0,051$ & $-0,103$ & 0,151 & 0,045 & $-0,019$ \\
\hline Tristeza & $-0,023$ & $-0,042$ & 0,117 & $-0,017$ & 0,012 \\
\hline Nervosismo & $-0,045$ & $-0,011$ & 0,040 & $-0,003$ & $-0,000$ \\
\hline Desânimo & 0,017 & $-0,019$ & 0,001 & 0,065 & 0,066 \\
\hline Com dúvidas & $-0,009$ & $-0,041$ & $-0,036$ & 0,003 & 0,027 \\
\hline Tédio & 0,032 & 0,003 & 0,010 & $-0,017$ & 0,022 \\
\hline Desesperança & $-0,000$ & 0,000 & $-0,000$ & 0,000 & $-0,000$ \\
\hline Raiva & 0,000 & $-0,000$ & 0,000 & 0,000 & 0,000 \\
\hline Sentiu mal & 0,000 & $-0,000$ & 0,000 & 0,000 & $-0,000$ \\
\hline
\end{tabular}

Método de extração dos fatores: análise dos componentes principais.

mas não esperançoso, ou então, não estava tranquilo, mas esperançoso. Os outros fatores revelaram uma variação total fraca, abaixo de 11\%, não sendo considerados para análise.

A contraposição das emoções de esperança e tranquilidade no segundo fator é explicada pela psicobiologia e pela análise do comportamento. A emoção refere-se a reações psicomotoras e alterações neurovegetativas em resposta a um estímulo ambiental. No caso da ansiedade, define-se como um estado de apreensão ou tensão, acompanhado por sensações físicas, induzido por estímulos ameaçadores (expectativa de perigo ou de dor) (Brandão, 2004); isto é, a ansiedade depende da apresentação de estímulos aversivos ou remoção de reforçadores positivos. Por outro lado, a elação (alegria) é gerada pela obtenção de reforçadores positivos (Catania, 1999). Considerando a esperança um conjunto de sentimentos referentes à expectativa de obtenção de reforçadores positivos e a tranquilidade o oposto de ansiedade (remoção de reforçadores positivos), é de se esperar que nesta condição de vivência de cirurgia cardíaca (estímulo aversivo) os pacientes expressem esta contraposição. Eles apresentaram espe- rança (de resgatar a saúde e sobreviver - reforçador positivo), mas, em contrapartida, estavam ansiosos (ou seja, não estavam tranquilos) pela possibilidade de riscos que a cirurgia envolve (perder a vida - remoção de reforçador positivo). Esta análise de contingências é fundamental para o planejamento da intervenção psicológica. A identificação dos fatores relacionados à resposta emocional e a especificação dos componentes desta resposta guiam a tomada de decisão do profissional quanto ao método de atuação mais adequado a ser empregado. Baseando-se nos dados coletados, o psicólogo levanta hipóteses e atua de forma a mudar as variáveis situacionais para favorecer mudanças no comportamento (Gorayeb \& Guerrelhas, 2003).

Embora os resultados tenham elucidado alguns aspectos do impacto psicológico de um grupo educativo para pacientes de cirurgia cardíaca, a generalização dos resultados é questionada, uma vez que eles foram analisados considerando os pacientes como um grupo homogêneo, ao invés de distingui-los entre aqueles que estavam no período pré-operatório e outros que já tinham sido submetidos à cirurgia. Possivelmente, as duas categorias estiveram expostas a estímu- 
los ambientais diferenciados ao longo do tratamento, os quais eliciaram ou favoreceram distintas respostas. Logo, é de se esperar uma variação entre pacientes do período pré e pós-operatório quanto à reação ao grupo educativo. A variação entre os grupos só é conhecida se a avaliação discernir o perfil dos pacientes. Deve-se salientar que, dada a complexidade inerente ao tema, apesar das limitações metodológicas, a pesquisa fomentou discussões importantes sobre técnicas de intervenção em saúde, sobre o papel do psicólogo na equipe interdisciplinar e sobre as mudanças institucionais necessárias para a efetivação de um trabalho pautado no paradigma da Promoção de Saúde.

\section{Conclusão}

O estudo alcançou o objetivo de avaliar o impacto psicológico de um grupo educativo de cirurgia cardíaca em pacientes de um hospital universitário. Verificou-se que o grupo foi benéfico para o ajustamento psicossocial dos pacientes ao estressor, pois eles se sentiram esperançosos após participarem da intervenção e avaliaram que a atividade foi muito importante para ajudar a enfrentar o procedimento cirúrgico.

O método de atuação no grupo educativo foi fundamentado em uma abordagem pedagógica tradicional e centralizada no discurso de culpabilidade do sujeito pela condição de adoecimento. A reestruturação do programa é necessária, sugerindo-se o incremento das estratégias didáticas e, em um nível mais abrangente, reformulação dos objetivos da atividade a partir do paradigma da Promoção de Saúde, almejando não somente o repasse de informações, mas também o desenvolvimento no paciente de habilidades de autoconhecimento e autogestão.

A aplicação dos preceitos de Promoção de Saúde na reformulação do programa educativo requer implementação de mudanças institucionais. Neste sentido, o psicólogo deve exercer a tarefa de analista institucional, que é identificar a estrutura de organização hospitalar e os obstáculos às mudanças. Feito isto, o esforço é direcionado para motivar a equipe a realizar um movimento institucionalista de modificação cultural, voltado para a compreensão do paciente como um indivíduo capaz de aprender melhores formas de enfrentar os problemas de adoecimento e de desenvolver comportamentos

38 saudáveis independentemente.
O programa educativo por si só foi insuficiente para minimizar totalmente o sentimento de medo da cirurgia. Outras estratégias de intervenção direcionadas para modificação das respostas emocionais precisam ser planejadas e implementadas pela equipe interdisciplinar. O psicólogo pode contribuir ao executar uma análise funcional das contingências às quais o paciente está exposto e programar, junto à equipe, ações direcionadas para a mudança de fatores situacionais relacionados à resposta emocional. Desta forma, a prática deve envolver não somente aplicação de técnicas psicológicas, mas também reestruturação das contingências de assistência em saúde.

Falhas metodológicas limitaram o estudo, dificultando a generalização dos resultados. Futuras investigações devem primar pelo rigor na construção do instrumento e pela precisão na análise dos dados. No entanto, o estudo contribuiu para acrescentar conhecimentos científicos à Psicologia da Saúde no que tange à investigação do impacto psicológico de uma intervenção educativa e, em termos práticos, tratou do envolvimento do psicólogo em ações de adequação dos serviços hospitalares ao paradigma da Promoção de Saúde.

\section{Referências}

Alfonso, L. M., \& Abalo, J. A. G. (2004). La investigación de la adherencia terapéutica como un problema de la psicología de la salud. Psicología Y Salud, 14 (1), 89-99.

Angerami-Camon, V. A. (Org.) (1996). O doente, a psicologia e o hospital (3a. ed.). São Paulo: Pioneira.

Bandura, A., Azzi, R. G., \& Polydoro, A. (Coords.). (2008). Teoria social cognitiva: conceitos básicos. Porto Alegre: Artmed.

Benute, G. R. G., Santos, N. O., Santos, R. M. R., Rondon, F. C., Quayle, J. M. B. R., \& De Lucia, M. C. S. (2001). A importância do psicólogo na criação e implantação dos programas educativos e de prevenção em saúde. Revista Brasileira de Educação Médica, 25 (1), 49-53.

Brandão, M. L. (2004). As bases biológicas do comportamento: introdução à neurociência. São Paulo: EPU.

Baremblitt, G. (2002). Compêndio de análise institucional e outras correntes: teoria e prática (5a. ed.). Belo Horizonte: Instituto Felix Guattari.

Catania, A. C. (1999). Aprendizagem comportamento, linguagem e cognição. Porto Alegre: Artmed.

Coates, V. (1999). Education for patients and clients. London: Taylor \& Francis. 
Crivisqui, E. (1999). Apresentação da análise em componentes principais (ACP). Londrina: UEL.

Cruz, C., \& Ribeiro, U. (2003). Metodologia científica: teoria e prática. Rio de Janeiro: Axcel Books.

Fernandes, A. C. V. L., \& Mclntyre, M. T. M. (2006). Intervenção multimodal em pacientes na reabilitação pós-enfarte do miocárdio em Portugal. Revista Brasileira em Promoção da Saúde, 19 (2), 74-83.

Gorayeb, R., \& Guerrelhas, F. (2003). Sistematização da prática psicológica em ambientes médicos. Revista Brasileira de Terapia Comportamental e Cognitiva, 5 (1), 11-19.

Granada, G. G. (2004). Grupos educativos multiprofissionais e promoção à saúde: a experiência em um centro de saúde. Dissertação de mestrado não-publicada, Universidade Estadual de Campinas.

Kerbauy, R. R. (2002). Comportamento e saúde: doenças e desafios. Psicologia USP, 13 (1), 11-28.

Lazarus, R. S. (1993). Coping theory and research: past, present, and future. Psychosomatic Medicine, 55 (3), 234-247.

Lipp, M. E. N. (2001). Treino psicológico de controle do stress como prática clínica para a redução na reatividade cardiovascular de hipertensos. Revista Temas em Psicologia: Sociedade Brasileira de Psicologia, 9 (2), 91-98.

López, M. P. S., García, M. E. A. \& Dresch, V. (2006). Ansiedade, autoestima y satisfacción autopercebida como preditores de la salud: diferencias entre hombres y mujeres. Psicothema, 18 (3), 584-590.

Maia, S. M. (2006). Ainterconsulta psicológica no hospital geral: uma demanda institucional. Dissertação de mestrado nãopublicada, Universidade Federal de Minas Gerais, Belo Horizonte.

Medeiros, V. C. C. (2002). Paciente cirúrgico: a influência da ansiedade nas estratégias de enfrentamento utilizadas no período pré-operatório. Dissertação de mestrado nãopublicada, Universidade de São Paulo.

Neme, C. M. B., \& Rodrigues, O. M. P. R. (Orgs.) (2003). Psicologia da saúde: perspectivas interdisciplinares. São Carlos: Rima.
Pereira, A. L. F. (2003). As tendências pedagógicas e a prática educativa nas ciências da saúde. Cadernos de Saúde Pública, 19 (5), 1527-1534.

Resende, M. C., Cunha, C. P. B., Silva, A. P., \& Sousa, S. J. (2007). Rede de relações e satisfação com a vida em pessoas com amputação de membros. Ciências e Cognição, 10, 164-172.

Ribeiro, J. L. (2004). Avaliação das intenções comportamentais relacionadas com a promoção e protecção da saúde e com a prevenção de doenças. Análise Psicológica, 2 (22), 387-397.

Romano, B. W. (Org.) (2001). Psicologia ecardiologia:encontros possíveis. São Paulo: Casa do Psicólogo.

San Gregorio, M. A. P., Rodríguez, A. M., \& Rodríguez, A. G. (2005). Problemas psicológicos asociados al transplante de órganos. International Journal of Clinical and Health Psychology, 5 (1), 99-114.

Scorsafava, A. T. (2004). A estratégia de saúde da família como novo campo de atuação para o psicólogo: o modelo de Sobral/CE. Monografia de especialização não-publicada, Escola de Formação em Saúde da Família Visconde de Sabóia.

Seidl, E. M. F., Tróccoli, B. T., \& Zannon, C. M. (2001). Análise fatorial de uma medida de estratégias de enfrentamento. Psicologia: Teoria e Pesquisa, 17 (3), 225-234.

Souza, R. H. S. (2004). Sentimentos e percepções do cliente no pré-operatório de cirurgia cardíaca. Dissertação de mestrado não-publicada, Universidade Federal do Paraná, Curitiba.

Tonetto, A. M., \& Gomes, W. B. (2007). A prática do psicólogo hospitalar em equipe multidisciplinar. Estudos de Psicologia (Campinas), 24 (1), 89-98.

Recebido em: 29/4/2008

Versão final reapresentada em: 12/12/2008

Aprovado em: 7/5/2009 